\title{
Modeling the Short-Term Unfairness of IEEE 802.11 in Presence of Hidden Terminals
}

\author{
Zhifei $\mathrm{Li}^{1}$, Sukumar Nandi ${ }^{2}$, and Anil K. Gupta ${ }^{1}$ \\ 1 School of Computer Engineering, Nanyang Technological University, \\ Singapore, 639798 \\ \{pg03802331, asgupta\}@ntu.edu.sg \\ 2 Dept. of Computer Science \& Engineering, Indian Institute of Technology, \\ Guwahati, India, 781039 \\ sukumar@iitg.ernet . in
}

\begin{abstract}
In this paper, using a simple hidden-terminal scenario, we show that IEEE 802.11 exhibits substantial short-term unfairness, though it provides long-term fairness. We analyze the short-term behavior using embedded-Markov chain method to answer the following two questions: (i) once a node gets control of the medium, what is the average number of packets this node can transmit consecutively without experiencing any collision, (ii) once a node loses its control of the medium, what is the average time the node has to wait before it gets control of the medium again. The first question reflects on how long a node can capture the medium, whereas the second question reflects on how long a node may be starved. The analytical model is validated by the simulation results. Our work is distinct from most of the work published in the literature in two aspects: we focus on the short-term behavior rather than the long-term, and the analytical method is adopted for the study.
\end{abstract}

\section{Introduction}

IEEE 802.11 [6] is the de facto standard for Wireless LANs, and it defines two MAC protocols: Point Coordination Function (PCF) and Distributed Coordination Function (DCF). However, only the DCF is popular. As DCF operates in a distributed manner, achieving fairness in accessing the medium is one of the most challenging issues. Based on the length of the time over which we observe the system, the fairness can be defined on a short-term basis and a long-term basis. The short-term fairness automatically gives rise to long-term fairness, but not the vice versa [7]. In particular, under certain scenarios, though the bandwidth allocation is fair in a long-term, it is very unfair if we view the system from a short-term viewpoint. The short-term fairness is important for the adaptive traffic (e.g., TCP traffic) and for the delay- or jitter-sensitive traffic [7]. In this paper, we aim to analyze the short-term unfairness of IEEE 802.11.

The duration, over which the short-term fairness should be measured, is difficult to define as it depends upon the requirements of applications as well as upon the channel bandwidth. To get around this problem, Jain's index [7] can 
be used to reflect fairness over different time scales. Though the index is useful in comparing fairness of two different protocols, the absolute value of the index for a given protocol does not express the fairness of the protocol very clearly. Therefore, we measure the short-term fairness in an alternate way by evaluating the following two metrics: (i) once a node gets control of the medium, what is the average number of packets this node can transmit consecutively without experiencing any collision, (ii) once a node loses its control of the medium, what is the average time the node has to wait before it gets control to the medium again.

In this paper, using an embedded Markov chain model, the above two metrics are measured based on the concepts of 'expected state holding time' and 'expected first passage time'. The analytical model is validated by the simulation results. Our results show that the IEEE 802.11 exhibits substantial short-term unfairness even in a very simple hidden-terminal scenario.

The remainder of the paper is organized as follows. In Section 2, using simulation method, we show that IEEE 802.11 exhibits considerable short-term unfairness in the hidden-terminal scenario. In Section 3, the Markov chain model is described. Section 4 presents the analytical and simulation results. Related work is reviewed in Section 5 and the paper is concluded in Section 6.

\section{Short-Term Unfairness in IEEE 802.11}

To show the short-term unfairness in IEEE 802.11, we simulate the well-known hidden-terminal scenario depicted in Figure 1. There are three nodes, A, B and C, with two single-hop flows: flow from A to B, and flow from C to B. Since nodes $\mathrm{A}$ and $\mathrm{C}$ cannot hear from each other, they may simultaneously try to communicate with a common node, i.e., node B, resulting in a collision. In such a situation, nodes $\mathrm{A}$ and $\mathrm{C}$ are referred as the hidden terminals of each other.

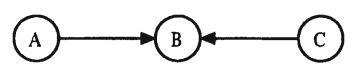

Fig. 1. Hidden-terminal Scenario

In the simulation, NS-2 with CMU wireless extensions [4] is used. For each single-hop flow, a Constant Bit Rate (CBR) traffic is adopted, where each packet is 1000-bytes long. The raw bandwidth is set with $2 \mathrm{Mbps}$, and the maximum throughput is about 1.4 Mbps due to the overhead of IEEE 802.11. The source rate of each single flow is made greater than the medium capacity, since unfairness occurs only when the system is overloaded.

The simulation results show that each flow gets an average throughput of about $0.7 \mathrm{Mbps}$, indicating that the two flows share the medium fairly on a long-term basis. However, if we compute the values of the two short-term metrics defined in Section 1, the protocol exhibits substantial unfairness. For metric (i), 
we find that, on an average, once a node gets control of the medium, it can transmit about 6.4 packets consecutively without collision. For the metric (ii), once a node (say node $\mathrm{C}$ ) loses control of the medium, it has to wait for the other node (i.e., A) to transmit about 27 packets before it gets control of the medium again. However, this does not mean that node A can transmit 27 packets consecutively without collision. We illustrate this using the following example. Consider that after node $\mathrm{C}$ loses control of the medium, node A gets control of the medium and it transmits one or more packets consecutively. Then, one or more collisions occur. After the collision(s), node A again gets control and transmits one or more packets consecutively. In addition to the average values, we also observed the corresponding maximum values, which are equal to 35 and 160 , respectively, for the two metrics. This shows how much short-term unfairness is ingrained in IEEE 802.11, which is unacceptable for jitter-sensitive traffic. In this section, we explain how hidden-terminal problem causes short-term unfairness.

\subsection{Basic Techniques in IEEE 802.11}

IEEE 802.11 adopts the well-known binary exponential back-off (BEB) algorithm as its contention resolution mechanism, which is described as follows. Every node maintains a Contention Window (CW) and a back-off timer. Before every transmission, the node first defers by a back-off timer, which is generated according to equation (1), unless the back-off timer already contains a non-zero value, in which case it is unnecessary to generate a new random back-off timer.

$$
\text { BackoffTime }=\text { Random }() \times \text { SlotTime }
$$

The SlotTime is specified by the physical layer, and the random value is uniformly distributed over the range $[0, \mathrm{CW}]$. For the first transmission attempt of a packet, the $\mathrm{CW}$ is set to $C W_{\min }$. Whenever a retransmission is initiated, the $\mathrm{CW}$ is doubled until the $C W_{\max }$ is reached. After that, the $\mathrm{CW}$ remains at the maximum value until the retry limit (say, $n$ ) is reached. Once the retry limit is reached, the $\mathrm{CW}$ will be reset to $C W_{\min }$. The $\mathrm{CW}$ is also reset to $C W_{\text {min }}$ whenever a transmission attempt is successful. For the convenience, we call each retransmission attempt as a stage, whose number is in the range $[0, n-1]$.

To combat with the hidden-terminal problem, IEEE 802.11 defines a fourway handshake, where a sequence of Request To Send (RTS), Clear To Send (CTS), Data, and ACK frames, is transmitted for the transmission of every single data packet. For the convenience, we call the exchange of RTS/CTS/Data/ACK frames as a frame exchange sequence (FES). Moreover, FES $(X, Y)$ represents a FES between nodes $\mathrm{X}$ and $\mathrm{Y}$, initiated by node $\mathrm{X}$, implying node $\mathrm{X}$ sends one packet successfully to node $\mathrm{Y}$. Moreover, we call node $\mathrm{X}$ as the transmitting node and $\mathrm{Y}$ as the receiving node, while all the other nodes are called the waiting nodes. Note that the word 'packet' implies the protocol data unit (PDU) of a higher layer whereas 'frame' is the MAC layer PDU.

When a FES is in progress, the waiting node freezes its back-off timer. After the FES is successfully completed, all the nodes first defer for a DCF Inter-Frame 
Space (DIFS) period. Then, the transmitting node generates a new random value from its $\mathrm{CW}$ and backs-off before it initiates another FES. On the other hand, the waiting node simply resumes to count down from its frozen backoff timer. It is easy to see that the transmitting node may transmit several packets consecutively before the waiting node's back-off timer is reduced to zero. Contrary to a successful transmission, when a collision occurs, all the colliding nodes will generate a new random value from their corresponding CWs.

\subsection{Explanation for the Short-Term Unfairness}

In the four-way handshake, once the RTS/CTS has been completed successfully, the hidden-terminal problem may not arise any more. For example, in Figure 1, once node B sends back a CTS to node A, node C overhears this CTS and thus defers its transmission, avoiding collision. The four-way handshake solves the hidden-terminal problem largely by introducing the RTS/CTS handshake before the real Data frame is transmitted. However, it cannot eliminate the problem completely as RTS/CTS cannot always be transmitted successfully.

Now let us derive the condition under which the RTS/CTS can be successful when two hidden nodes ( $\mathrm{A}$ and $\mathrm{C}$ ) contend for the medium (Figure 1). The condition is as follows: after a collision or a FES, the difference between the back-off timers at the two hidden nodes should be large enough for node $\mathrm{B}$ to send back a CTS to node A (C) before that node C (A) starts sending its RTS. The minimum time difference required is equal to the transmission time of RTS plus a Short Inter-Frame Space (SIFS). This can be expressed as:

$$
|Z|>\text { Len }=\text { TxTime }(R T S)+S I F S
$$

where $Z$ is the difference between the back-off timer. Len is equal to about 19 slots when the slot time is $20 \mu s$ for DSSS [6]. It is easy to see that the condition is difficult to satisfy when the CWs at the contending nodes are small (e.g., 31).

Now let us explain how the hidden-terminal problem causes short-term unfairness. Consider the situation that the CWs at nodes $\mathrm{A}$ and $\mathrm{C}$ are very small (e.g., 31). As discussed above, under such situation, the transmission of RTSs of nodes $\mathrm{A}$ and $\mathrm{C}$ may overlap partially, and as a result collide. The collision may occur several times until the CWs are large enough to allow either node (say, node A) to get control of the medium. Once the FES (A, B) is completed, node $\mathrm{A}$ resets its $\mathrm{CW}$ to $C W_{\min }$ and backs-off before initiating another FES. However, the remaining back-off timer at node $\mathrm{C}$ may be large compared to the back-off timer at node $\mathrm{A}$, and thus nodes $\mathrm{A}$ and $\mathrm{B}$ may exchange several more FESs before node C's back-off timer reduces to a small value.

Whenever the back-off timer at node $\mathrm{C}$ reduces to a small value, node $\mathrm{C}$ contends for the medium. However, as the $\mathrm{CW}$ at node $\mathrm{A}$ is equal to $C W_{\min }$, the contention is most likely to result in a collision again. After the collision, node $\mathrm{A}$ doubles its $\mathrm{CW}$ from $C W_{\min }$ whereas node $\mathrm{C}$ doubles its $\mathrm{CW}$ from a larger value (at least 63). Therefore, the $\mathrm{CW}$ at node $\mathrm{C}$ is greater than that at $\mathrm{A}$, and node $\mathrm{A}$ is more likely to get control of the medium again. Moreover, 
this process (i.e., several packet transmissions by node A, followed by collisions, and then transmissions by node $\mathrm{A}$ again) may repeat several times, leading to starvation at node $\mathrm{C}$ for a long period (compared to the time needed for a FES).

However, several mechanisms incorporated in IEEE 802.11 prevent node $\mathrm{C}$ from starving completely, such as: (i) after every FES, node A will back-off before initiating another FES, which gives node $\mathrm{C}$ a chance to contend for the medium with node $\mathrm{A}$; (ii) the $\mathrm{CW}$ at node $\mathrm{C}$ will be reset to $C W_{\min }$ after the retry limit $n$ is reached. Once node $\mathrm{C}$ controls the medium, it can transmit consecutively in a similar way, and thus the long-term fairness between the two flows is ensured.

\section{Analytical Modeling}

In this section, we model the hidden-terminal scenario in Figure 1 using an embedded Markov chain.

\subsection{Markov Chain Model}

At any point of time, the medium is in one of the following five states: $T_{A}$, $T_{B}, T_{C}, C o l$ and $I d l e$, where $T_{A}$ means that node $\mathrm{A}$ is getting the control of the medium and transmitting its packet, i.e., $\operatorname{FES}(\mathrm{A}, \mathrm{B})$ is in progress. Similarly, $T_{B}$ and $T_{C}$ correspond to nodes $\mathrm{B}$ and $\mathrm{C}$, respectively. However, in our considered scenario, $T_{B}$ does not arise, as node $B$ does not have any data packets to send. State $\mathrm{Col}$ means that there is a collision on the medium, while state Idle means that there is no transmissions or collisions over the medium. As our objective is to analyze the fairness rather than the capacity utilization of the medium, we do not need to consider the Idle state. As a result, only three states of the medium are considered: $T_{A}, T_{C}$, and Col. When the medium is either in $T_{A}$ or in $T_{C}$, we simply say that the medium is in a $T$ state. Since the transition probabilities among these three states depend on the values of CWs at nodes $\mathrm{A}$ and $\mathrm{C}$, which, in turn, are determined by the corresponding stages, the system can be modeled using three random variables: state of the medium, stage at node $\mathrm{A}$, and stage at node $\mathrm{C}$. Therefore, the system states are $\left(T_{A}, k, l\right),\left(T_{C}, k, l\right)$, and $(C o l, k, l)$, where $k$ and $l$ denote the stages at nodes $\mathrm{A}$ and $\mathrm{C}$, respectively. Obviously, $k, l=0, \ldots,(n-1)$. Note that we must use stages rather than the values of $\mathrm{CW}$ to represent the system state, since once the CW reaches the maximum value, it remains unchanged before resetting. When the medium is in state $T_{A}$, it is easy to see that the stage at the transmitting node (i.e., node A) must be zero, that is, only $\left(T_{A}, 0, l\right)$ system states are possible. Similarly, only $\left(T_{C}, k, 0\right)$ system states are possible. Therefore, if the retry limit is $n$, there are $n^{2}$ number of $C o l$ states, and $n$ number of states corresponding to each of $T_{A}$ and $T_{C}$, and thus the number of all possible system states is:

$$
N_{\text {state }}=n^{2}+2 n
$$

From a Col state, whenever a transition occurs, the system can enter anyone of the three kinds of states as shown in the leftmost diagram of Figure 2. If the 
next state is also a $C o l$ state, both of the stages, $k$ and $l$, are incremented by one, except that the stage is reset to zero whenever the retry limit $n$ is reached. On the other hand, if the next state is a $T$ state, the stage at the transmitting node is reset while the other stage remains unchanged.

In state $T_{A}$, whenever node $\mathrm{A}$ transmits another packet, it is natural to view this event as a self-transition. However, if we model the system in such a way, the transition probabilities depend upon the remaining back-off timer at the waiting node (i.e., node $\mathrm{C}$ ), which in turn, depends upon how many times this timer has been frozen, i.e., how many self-transitions have occurred in the $T_{A}$ state. This requires memorizing the history to obtain the current transition probabilities, which violates the memoryless requirement of a Markov chain. Therefore, we do not treat this event as a state transition. Rather, whenever in state $T_{A}$, a state transition occurs only when the system enters a $\mathrm{Col}$ state or a $T_{C}$ state. Therefore, whenever the system enters a $T_{A}$ state, the time that the system will remain in that state depends on the number of packets that node A can transmit consecutively before that node $\mathrm{C}$ controls the medium or that a collision occurs. Similar explanation applies for the state $T_{C}$. The state transitions from a $T$ state are illustrated in the remaining diagrams of Figure 2. It is easy to see that the chain obtained in such a way is an embedded Markov chain, modelling the underlying semi-Markov process.
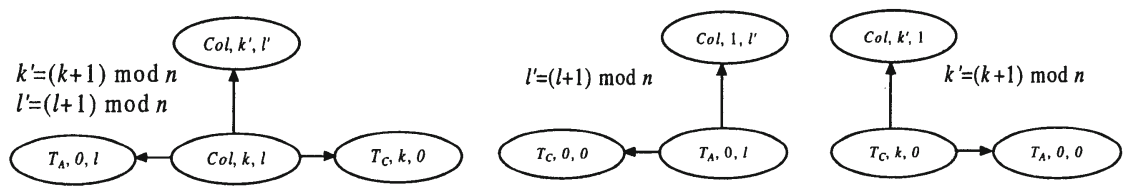

Fig. 2. State Transitions Diagrams

To illustrate the model clearly, we present the complete state transition diagram in Figure 3, where we assume $n=3$.

\subsection{Basic Analysis of the Model}

For the convenience, rather than representing the system states using three variables as above, we assign a single variable to represent the states, by ordering them as indicated in Table 1.

For the embedded Markov chain, we need to know the transition probability matrix, $P$. Once we get the matrix $P$, we can find the steady state probability vector, $\pi$, by solving the following equation:

$$
\left\{\begin{array}{l}
\pi=\pi \cdot P \pi=\left[\pi_{0}, \pi_{1}, \pi_{2}, \ldots\right], P=\left[p_{i j}\right] \\
\sum_{i} \pi_{i}=1 i \in\left[0, n^{2}+2 n-1\right]
\end{array}\right.
$$

In a discrete Markov chain, if the interval between two consecutive transitions (including self-transition) is identical, the steady state probability $\pi_{i}$ reflects the 


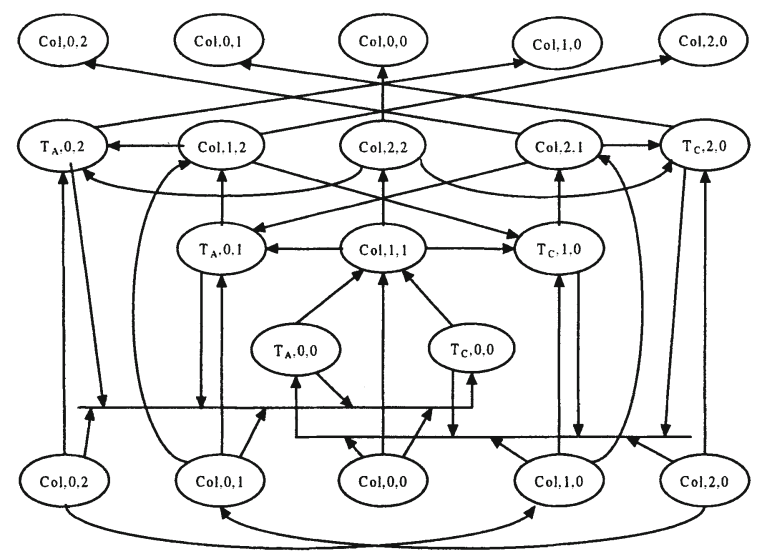

Fig. 3. State Transition Diagram for $n=3$

Table 1. Re-designating the State Variables

\begin{tabular}{|c|c|}
\hline States & Single Variable Range \\
\hline$\left(T_{A}, 0,0\right)$ to $\left(T_{A}, 0, n-1\right)$ & {$[0, n-1]$} \\
\hline$\left(T_{C}, 0,0\right)$ to $\left(T_{C}, n-1,0\right)$ & {$[n, 2 n-1]$} \\
\hline$(C o l, 0,0)$ to $(C o l, n-1, n-1)$ & {$\left[2 n, n^{2}+2 n-1\right]$} \\
\hline
\end{tabular}

proportion of the time that the system is in state $i$. However, this is not true in our model. For example, the interval between a $T$ state and its next state is a random variable. Therefore, $\pi_{i}$ can only tell us the probability that the system enters state $i$ whenever a transition occurs [5]. To get the time average state probability of being in state $i$, we must first analyze the holding time of state $i$.

Let $\mu_{j i}$ denote the average time that the system will remain in state $i$ once a transition from state $j$ to $i$ occurs. The $\mu_{j i}$ is given by:

$$
\mu_{j i}= \begin{cases}0 & p_{j i}=0 \\ 1 & p_{j i}>0 \& i \in\left[2 n, n^{2}+2 n-1\right] \\ r \times \operatorname{num}(j, i) & p_{j i}>0 \& i \in[0,2 n-1]\end{cases}
$$

Whenever the transition probability $p_{j i}$ is zero, the $\mu_{j i}$ must also be zero. The holding time in a Col state, since it does not depend upon the previous or present state, is assigned a unit value as in the second line of the above formula. To express the holding time of a $T$ state, let us define $n u m(j, i)$, which denotes the average number of packets the transmitting node can transmit consecutively once the system reaches the $T$ state (i.e., state $i$ ) from state $j$. Clearly, the holding time of a $T$ state is proportional to $\operatorname{num}(j, i)$. Moreover, we define the 'FES time' as the time needed for a FES to be completed, and 'Col time' as the time needed to detect the collision, while $r$ is the ratio between the 'FES time' and the 'Col time'. The 'Col time' is corresponding to the CTSTimeout 
interval defined in [6], which is independent from the length of the Data frame. Therefore, whenever the system enters a $T$ state, the average holding time can be represented by $r \times n u m(j, i)$, if the time required to detect a collision is unity. This explains the last line of equation (5). From $\mu_{j i}$, which corresponds to a transition, we can get $\mu_{i}$, the expected holding time of state $i$, as follows:

$$
\mu_{i}=\sum_{j} \frac{\pi_{j} \times p_{j i}}{\pi_{i}} \mu_{j i}
$$

It is easy to see that the $\mu_{i}$ corresponding to a $\mathrm{Col}$ state is always equal to one. Corresponding to a $T$ state, we define num $(i)$ in equation (7), which denotes the average number of packets the transmitting node can transmit consecutively once the system reaches state $i$.

$$
\operatorname{num}(i)=\sum_{j} \frac{\pi_{j} \times p_{j i}}{\pi_{i}} n u m(j, i) \quad i \in[0,2 n-1]
$$

As a result, formula (6) can be replaced by:

$$
\mu_{i}=\left\{\begin{array}{l}
n u m(i) \times r i \in[0,2 n-1] \\
1 \quad i \in\left[2 n, n^{2}+2 n-1\right]
\end{array}\right.
$$

Now we can get $\rho_{i}$, which represents the time average state probability of state $i[5]$. Note that here we have ignored the time the meidum being idle.

$$
\rho_{i}=\frac{\pi_{i} \times \mu_{i}}{\sum_{j} \pi_{j} \times \mu_{j}} ; i, j \in\left[0, n^{2}+2 n-1\right]
$$

\subsection{Derivation of the Metrics}

We now obtain the two metrics defined in Section 1. To recall, the first metric is, once a node gets control of the medium, what is the average number of packets this node can transmit consecutively without any collision. In our Markov chain model, let us say for node A, it is not possible for the system to travel from one $T_{A}$ state to another $T_{A}$ state without visiting a $T_{C}$ or $\mathrm{Col}$ state. Moreover, once the system enters a given $T_{A}$ state (let us say, state $i$ ), the average number of units of 'FES time' for which the system remains in that $T_{A}$ state, is simply equal to $\operatorname{num}(i)$, which is defined by equation (7). Therefore, the metric-1 can be obtained by taking average of all num(i) corresponding to $T_{A}$ states:

$$
H_{\text {metric-1 }}=\sum_{i=0}^{n-1} \frac{\pi_{i}}{\pi(A)} \times n u m(i) ; \pi(A)=\sum_{i=0}^{n-1} \pi_{i}
$$

Since the behavior is identical at nodes A and $\mathrm{C}$, the metric obtained for node $\mathrm{A}$ are also applicable to node $\mathrm{C}$.

Now, we recall that the second metric is, once a node loses its control of the medium, what is the average time the node has to wait before it gets control to the medium again. Since there are only two nodes (i.e., A and C) contending for the medium (Figure 1), the metric, let us say for node $\mathrm{C}$, can be replaced by: once the medium is controlled by node $\mathrm{A}$, what is the average number of packets that 
A can transmit before the medium is controlled by node C. This simply implies that once the system enters a $T_{A}$ state, what is the average number of units of ' $F E S$ time' that the system can remain in any $T_{A}$ state (via visiting $C o l$ state in-between) before the system enters a $T_{C}$ state. Note that the second metric allows a visit to a $\mathrm{Col}$ state in-between two $T_{A}$ states, which is not permitted in the first metric.

Here we use the concept of "expected first passage time" [5], which means that if the system starts in a given $T_{A}$ state (let us say, state $i$ ), what is the expected time after which the system will enter any $T_{C}$ state for the first time. The expected first passage time, $V_{i}$, can be expressed as follows [5]:

$$
V_{i}=\left\{\begin{array}{lr}
0 & i \in[n, 2 n-1] \\
R_{i}+\sum_{j} p_{i j}^{\prime} V_{j} & i \in[0, n-1] \cup\left[2 n, n^{2}+2 n-1\right]
\end{array}\right.
$$

where $R_{i}$ is the immediate reward once the system enters state $i$. For a $T_{A}$ state, it is equal to the corresponding num(i). Since all $T_{C}$ states are the trapping states, $R_{i}$ is zero for these states [5]. We should also assign $R_{i}$ with zero for all $\mathrm{Col}$ states, as our objective is to obtain the number of FESs, rather than the number of collisions. Therefore,

$$
R_{i}= \begin{cases}\operatorname{num}(i) & i \in[0, n-1] \\ 0 & i \in\left[n, n^{2}+2 n-1\right]\end{cases}
$$

In equation (11), $p_{i j}^{\prime}$ is a modified value of the transition probability $p_{i j}$. Since the $T_{C}$ states are considered as trapping states, the transition probabilities out of a $T_{C}$ state is set to zero, whereas the self-transition probability for each $T_{C}$ state is set to one. Other transition probabilities remain unchanged. Therefore,

$$
p_{i j}^{\prime}= \begin{cases}0 & i \in[n, 2 n-1] \& j \neq i \\ 1 & i \in[n, 2 n-1] \& j=i \\ p_{i j} & i \in[0, n-1] \cup\left[2 n, n^{2}+2 n-1\right]\end{cases}
$$

Obviously, $V_{i}$ corresponding to the trapping states (i.e., all $T_{C}$ states) should be zero. For all the other states, $V_{i}$ is equal to the 'immediate reward' $R_{i}$ plus the expected reward earned from whatever state is entered next. This explains the equation (11).

The metric- 2 can be obtained by taking average over all $V_{i}$ values corresponding to the $T_{A}$ states:

$$
H_{\text {metric }-2}=\sum_{i=0}^{n-1} \frac{\pi_{i}}{\pi(A)} \times V_{i} ; \pi(A)=\sum_{i=0}^{n-1} \pi_{i}
$$

To solve the above equations, we only need to know the transition probability matrix $P$ and the state holding time num $(j, i)$. The calculation of the above values is not trivial. However, due to space limitation, we do not present it here. Please refer to [8] for a detailed analysis. 


\section{Numerical Results}

Here we evaluate the equations derived in Section 3, and compare the analytical results with the simulation results. The simulation environment is the same as described in Section 2. The results correspond to the case when $C W_{\min }=31$, $L e n=19, C W_{\max }=1024$, and $n=7$, which are typically used in IEEE 802.11. Since our main objective is to analyze the behavior at $T$ states, and the behavior at nodes $\mathrm{A}$ and $\mathrm{C}$ is identical, we only present the results for $T_{A}$ states.

State Probabilities: Table 2 presents the values of $\pi_{i}$ and $\rho_{i}$. To recall, $\pi_{i}$ represents the proportion of transitions entering state $i$, while $\rho_{i}$ reflects the proportion of time spent in state $i$. The analytical results are quite close to the simulation results. We also notice that, though the sum of $\pi_{i}$ is quite small (about 0.24 ), the sum of $\rho_{i}$ is quite large (0.496). Since the $T_{C}$ states also have the same values, the total fraction amount of time spent in $T$ states is about 0.992 , implying that only a very small amount of time is spent in the large number of Col states. The reason is that the ratio (i.e., $r$ ) between the 'FES time' and the 'Col time' is very large (i.e., 20 in our case). This shows the advantage of using the short RTS/CTS frames before the transmission of the long Data frame.

Table 2. State Probabilities Comparison

\begin{tabular}{|c|c|c|c|c|c|c|c|c|c|}
\hline \multicolumn{2}{|r|}{ From } & $\left(T_{\lambda}, 0,0\right)$ & $\left(T_{A, 0}, 1\right)$ & $\left(T_{A}, 0,2\right)$ & $\left(T_{A, 0,3}\right)$ & $\left(T_{\left.A_{0}, 0,4\right)}\right.$ & $\left(T_{A} 0,5\right)$ & $\left(T_{A, 0}, 6\right)$ & Total \\
\hline \multirow{2}{*}{$\pi_{i}$} & Model & 0.025 & 0.031 & 0.037 & 0.038 & 0.037 & 0.035 & 0.032 & 0.236 \\
\hline & Simulation & 0.025 & 0.038 & 0.039 & 0.039 & 0.037 & 0.034 & 0.031 & 0.243 \\
\hline \multirow[b]{2}{*}{$P_{1}$} & Model & 0.008 & 0.014 & 0.025 & 0.046 & 0.088 & 0.165 & 0.150 & 0.496 \\
\hline & Simulation & 0.008 & 0.015 & 0.023 & 0.045 & 0.088 & 0.167 & 0.150 & 0.496 \\
\hline
\end{tabular}

Expected State Holding Time: Table 3 presents the results of num(i), which denotes the average number of packets node A can transmit consecutively once the system enters a given $T_{A}$ state (i.e., state $i$ ). We see that the results match very closely. As the stage at the waiting node (i.e., node $\mathrm{C}$ ) increases, the num(i) also increases, which indicates that it becomes more unfair for node $\mathrm{C}$.

Table 3. Expected Holding Time Comparison

\begin{tabular}{|c|c|c|c|c|c|c|c|}
\hline num(i) & $\left(T_{A}, 0,0\right)$ & $\left(T_{A}, 0,1\right)$ & $\left(T_{A}, 0,2\right)$ & $\left(T_{A}, 0,3\right)$ & $\left(T_{A}, 0,4\right)$ & $\left(T_{A}, 0,5\right)$ & $\left(T_{A}, 0,6\right)$ \\
\hline Model & 1.006 & 1.450 & 2.143 & 3.830 & 7.496 & 14.858 & 14.893 \\
\hline Simulation & 1.002 & 1.206 & 1.829 & 3.616 & 7.461 & 15.336 & 15.348 \\
\hline
\end{tabular}

Expected First Passage Time: Table 4 presents the results of $V_{i}$, which represents, if the system starts in a given $T_{A}$ state (i.e., state $i$ ), what is the expected number of FESs after which the system will enter any $T_{C}$ state for the 
first time. Again, the results obtained from the model are quite close to those from simulation. When the stage at the waiting node (i.e., node $\mathrm{C}$ ) increases (from 0 to 4 ), the $V_{i}$ also increases. However, when the stage at the node $\mathrm{C}$ further increases (i.e., from 4 to 5 , and then 6 ), the $V_{i}$ decreases. First, let us explain why the $V_{i}$ corresponding to the $\left(T_{A}, 0,6\right)$ state is small. We recall that $V_{i}$ is equal to the immediate reward (i.e., $n u m(i)$ ) plus the expected reward earned from whatever state is entered next. When the system departs from the $\left(T_{A}, 0,6\right)$ state, the system is likely to enter state $(C o l, 1,0)$ where the stage at node $\mathrm{C}$ has been reset. From this $\mathrm{Col}$ state, the system is more likely to enter a $T_{C}$ state, in comparison to, from other $\mathrm{Col}$ states. For instance, when the system transits to a $C o l$ state from the $\left(T_{A}, 0,5\right)$ state, the stage at node $\mathrm{C}$ will not be reset, and the probability of transiting to a $T_{C}$ state is small. This implies that after leaving $\left(T_{A}, 0,6\right)$ state, the expected reward earned from the future states is smaller in comparison to that after leaving the state $\left(T_{A}, 0,5\right)$. Therefore, the $V_{i}$ corresponding to $\left(T_{A}, 0,6\right)$ is small compared to the $\left(T_{A}, 0,5\right)$ state. Now, we explain why the $V_{i}$ corresponding to the $\left(T_{A}, 0,5\right)$ state is smaller than that for the $\left(T_{A}, 0,4\right)$ state. Since the state $\left(T_{A}, 0,6\right)$ is a future state of $\left(T_{A}, 0,5\right)$, a small $V_{i}$ for $\left(T_{A}, 0,6\right)$ will also affect the $V_{i}$ for the $\left(T_{A}, 0,5\right)$ state. However, the effect of the reset behavior decreases rapidly as the stage at the waiting node becomes smaller than 4 . From the above discussion, it is clear that the resetting $C W$ mechanism adopted in IEEE 802.11 improves the short-term fairness.

Table 4. Expected First Passage Time Comparison

\begin{tabular}{|c|c|c|c|c|c|c|c|}
\hline$V_{i}$ & $\left(T_{A}, 0,0\right)$ & $\left(T_{A}, 0,1\right)$ & $\left(T_{A}, 0,2\right)$ & $\left(T_{A}, 0,3\right)$ & $\left(T_{A}, 0,4\right)$ & $\left(T_{A}, 0,5\right)$ & $\left(T_{A}, 0,6\right)$ \\
\hline Model & 11.796 & 25.416 & 31.081 & 34.059 & 34.404 & 30.273 & 17.661 \\
\hline Simulation & 10.413 & 23.584 & 29.998 & 34.031 & 34.896 & 31.430 & 18.555 \\
\hline
\end{tabular}

Metrics: Table 5 presents the values of the two metrics defined earlier. We also present the corresponding maximum values. Again, the analytical results match the simulation results. Note that the values of the two metrics differ largely. The reason is that whenever the system departs from a $T_{A}$ state, the probability that the system enters a $C o l$ state is very large. After the collision(s), the system is more likely to enter another $T_{A}$ state (rather than a $T_{C}$ state) as the $\mathrm{CW}$ at node $\mathrm{A}$ is smaller than that at node $\mathrm{C}$. This may be repeated several times, resulting in such a large difference.

General Applications of the Model: The above results correspond to the case when $C W_{\min }=31$, Len $=19, C W_{\max }=1024$, and $n=7$. By varying these parameters, the short-term behavior for other scenarios can be obtained. For example, by making Len=TxTime(Data) $+S I F S$, we can model the two-way handshake in the presence of hidden terminals. On the other hand, by making $L e n=0$, we can model anyone of the two handshakes without hidden terminals. By varying $n$ and $C W_{\min }$, we can model different physical layers, such as FSSS, DSSS and 
Table 5. Comparison of the Metrics

\begin{tabular}{|c|c|c|c|c|}
\hline From & metric-1 & $\begin{array}{c}\text { Max } \\
\text { metric-1 }\end{array}$ & metric-2 & $\begin{array}{c}\text { Max } \\
\text { metric-2 }\end{array}$ \\
\hline Model & 6.683 & NA & 27.379 & NA \\
\hline Simulation & 6.413 & 35 & 27.090 & 160 \\
\hline
\end{tabular}

IR [6]. Moreover, since the model can predict the short-term behavior precisely, it would also predict the long-term behavior accurately.

\section{Discussion and Related Work}

\subsection{Future Work}

We have presented a novel embedded-Markov model to study the short-term unfairness in a simple 3-nodes hidden-terminal case. We are extending the model to a more general scenario. However, it is necessary to mention that the modelling process described in Section 3 is quite general for the study of short-term behavior, especially the adoption of the first passage time.

Another focus is to propose a solution to cope with the short-term unfairness problem. From the results, we have already seen that the resetting $C W$ mechanism improves the short-term fairness. Therefore, in addition to the standard resetting mechanism, we are of the opinion that the CW should also be reset whenever the short-term unfairness occurs that can be detected using dynamic measurements. Our preliminary results show that this method improves the fairness. However, the aggregate throughput may degrade.

\subsection{Related Work}

The fairness problem in wireless networks have been extensively addressed $[9,10$, 11]. However, most of these work do not consider the hidden-terminal problem explicitly. Also, they mainly consider long-term unfairness. More importantly, there is no analytical model predicting the unfairness in IEEE 802.11. In contrast, in this paper, we have developed an analytical model to explain and predict the short-term unfairness due to the hidden-terminal problem.

Several analytical models of IEEE $802.11[1,2,3]$ have studied the long-term behavior (i.e., capacity) by ignoring many details of the protocol and adopting simplified assumptions. For example, the model in [1] overlooks the resetting $C W$ mechanism and assumes a constant collision probability, which is clearly imprecise as shown in our results. While these models are able to predict the longterm behavior, they cannot be used to study the short-term behavior accurately because the required details are lost in their models. In contrast, we model the IEEE 802.11 in a detailed manner (e.g., by including the resetting mechanism and all the possible collision states) to predict the short-term fairness precisely. 
The authors of [7] have studied the short-term fairness by first developing two fairness metrics and then applying the metrics in analyzing two MAC protocols: CSMA/CA and ALOHA. Though IEEE 802.11 is mainly based on CSMA/CA, it has many other features, and thus [7] cannot apply to IEEE 802.11. Moreover, they have not considered the hidden-terminal problem and they mainly focus on developing general fairness metrics, which are different from our work.

\section{Conclusions}

In this paper, we have presented an Embedded-Markov chain model for IEEE 802.11. Our model is novel in that it predicts the short-term unfairness of IEEE 802.11 very precisely, which is not available in the literature. The key concepts used in the model include the 'state holding time' and the 'first passage time'. Our results show that IEEE 802.11 exhibits substantial short-term unfairness in presence of hidden terminals. One important implication of our results is that the resetting of the contention window may improve the short-term fairness.

\section{References}

1. G. Bianchi, "Performance Analysis of the IEEE 802.11 Distributed Coordination Function," IEEE Journal on Selected Area in Communications, March 2000, pp.535-547.

2. F. Cali, M. Conti, E. Gregori, "Dynamic Tuning of the IEEE 802.11 Protocol to Achieve a Theoretical Throughput Limit," IEEE/ACM Transactions on Networking, December 2000, pp.785-799.

3. H.S. Chhaya, S. Gupta, "Performance modeling of asynchronous data transfer methods of IEEE 802.11 MAC protocol," ACM Wireless Networks, 3, 1997, pp.217234 .

4. CMU Monarch Group. CMU Monarch Extensions to NS, http://www.monarch.cs.cmu.edu/.

5. R.G. Gallager, "Discrete Stochastic Processes," Kluwer Academic Publishers, Boston, 1996.

6. IEEE, "Wireless LAN Medium Access Control (MAC) and Physical Layer (PHY) specifications," IEEE 802.11 standards, June 1999.

7. C.E. Koksal, H. Kassab, H. Balakrishnan, "An Analysis of Short-Term Fairness in Wireless Media Access Protocols," in ACM SIGMETRICS, 2000.

8. Z.F. Li, S. Nandi, A.K. Gupta, "Modeling the Short-term Unfairness of IEEE 802.11 in Presence of Hidden Terminals," full version of the paper, avaliable at http://www.ntu.edu.sg/home5/pg03802331/.

9. H. Lou, S. Lu, V. Bharghavan, "A New Model for Packet Scheduling in Multi-hop Wireless Networks," in ACM MOBICOM, 2000.

10. T. Nandagopal, T. Kim, X. Gao, V. Bharghavan, "Achieving MAC Layer Fairness in Wireless Packet Networks," in ACM MOBICOM, 2000.

11. N.H. Vaidya, P. Bahl, S. Gupta, "Distributed fair scheduling in a wireless LAN," in ACM MOBICOM, 2000. 